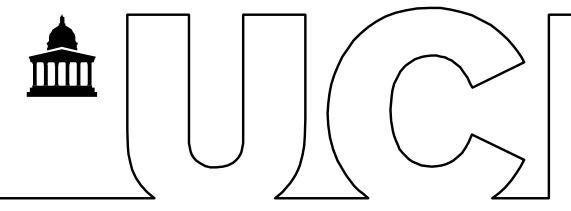

El-Turki, A; Long, P; Wong, ICK; Smith, H; (2016) How valid are IMS DA summary statistics of children's vaccination status? Pharmacoepidemiology and Drug Safety (In press). Downloaded from UCL Discovery: http://discovery.ucl.ac.uk/1508086.

\title{
ARTICLE
}

\section{How valid are IMS DA summary statistics of children's vaccination status?}

\author{
Aisha El-Turki ${ }^{1,2, *}$, Paul Long ${ }^{3}$, lan CK Wong ${ }^{1,4}$, Helen Smith ${ }^{2}$ \\ ${ }^{1}$ Centre for Paediatric Pharmacy Research, Research Department of Practice and Policy, \\ UCL School of Pharmacy, United Kingdom \\ ${ }^{2}$ Division of Primary Care \& Public Health, Brighton and Sussex Medical School, United \\ Kingdom \\ ${ }^{3}$ Institute of Pharmaceutical Science \& Department of Chemistry, King's College London, \\ London, United Kingdom \\ ${ }^{4}$ Centre for Safe Medication Practice and Research, Department of Pharmacology and \\ Pharmacy, Li Ka Shing Faculty of Medicine, The University of Hong Kong, Hong Kong, SAR, \\ China \\ * Corresponding author: Dr Aisha El-Turki, Division of Primary Care \& Public Health, \\ Brighton and Sussex Medical School, Mayfield House, Falmer, Brighton, BN1 9PH, United \\ Kingdom; Telephone: +44 (0)1273-641025; Fax: +44 (0)1273 644192; E-mail: a.el- \\ turki@bsms.ac.uk
}

Running head: Validation of children's vaccination status

Article key words: validation, children, pneumococcal conjugate vaccine, IMS DA, Key points:

- Accurate recording of immunization status is essential for any immunization program evaluation

- Historically IMS DA data has underestimated the pneumococcal vaccination rate in children

Abbreviations: PCV7- Seven-Valent Pneumococcal Conjugate Vaccination; IMS DA- IMS Disease Analyzer; COVER- Cover of Vaccination Evaluated Rapidly; GPs- General practitioners.

Word count: 2368

\begin{abstract}
Purpose: Accurate recording of immunisation status is essential for the evaluation of any immunization program. In September 2006, 7 Valent Pneumococcal Conjugate

Vaccination (PCV7) was introduced into the UK's routine childhood immunisation programme. This study validated the PCV7 immunization status of children aged 2 years recorded in the IMS Disease Analyses database.
\end{abstract}

Methods: The PCV7 vaccination uptake rate for children born in 2008 in the IMS DA database was calculated. A sample of 173 of the 2497 children not recorded as vaccinated was identified and a questionnaire was sent to each of their General Practitioners to ascertain the child's true PCV7 vaccination status.

Results: In the IMS DA data of 15,237 children born in 2008, 12,740 (83.6\%) had a vaccination record of PCV7. One hundred and eleven of the 167 questionnaires sent to the 
child's general practitioners were returned, giving an adjusted response rate of 111/167 $(66.5 \%)$. Based on the general practitioners' responses, $71(64 \%)$ of these children were fully vaccinated according to their General Practitioner's records making the revised estimated vaccination rate for this cohort $94.1 \%$

Conclusion: This validation study has shown that caution is needed if using historical IMS patient-level data to analyse the effectiveness of PCV7 as there is a potential underrecording of immunization leading to underrepresentation of vaccination rates by approximately ten per cent. Coverage of other vaccines may also be underestimated in the IMS database.

\section{Introduction}

Childhood immunization against infectious diseases is a key component of preventive health care. In September 2006, 7 Valent Pneumococcal Conjugate Vaccination (PCV7) was introduced into the UK routine childhood immunization programme at two and four months of age, with a booster dose after 12 months of age. ${ }^{1}$ To evaluate its impact and effectiveness on child health, it is important to have an accurate measure of uptake that can be used to investigate the association between the incidence of pneumococcal infections and PCV7 (Prevnar 7®) usage. One possible source of data on immunisation uptake is the IMS Disease Analyser (IMS DA). It is a private, international health care company which gathers anonymized data from a nationally representative sample of UK general practices. ${ }^{2}$ The data gathered from the sampled practices are used to calculate projected national vaccination coverage. In this study the accuracy of IMS-DA summary statistics of vaccination uptake was ascertained by comparing patient-level data from the database with the immunization status reported by the General Practitioner after reviewing the individual's health record.

\section{Methods}

Study design

This cross sectional observational study was designed to investigate the actual immunisation status of children with no record of having received PCV7 the IMS DA database.

\section{Data source}

The IMS Disease Analyzer (IMS-DA) (formally known as Mediplus) derives anonymised data from the electronic health records of approximately three million patient registered with over 500 general practitioners working in 125 general practices throughout the UK. Automatically downloaded data includes demographic characteristics, diagnoses, and prescriptions. Drugs are coded based on the Anatomical Therapeutic Chemical (ATC) classification and the Read codes used in UK General Practice for symptoms and diagnoses are mapped to ICD-10 (International Statistical Classification Disease, $10^{\text {th }}$ Revision) by the IMS Health team. ${ }^{3}$

\section{Source population}

The target population were children born in 2008. A list was compiled of children who by the age of two had no record of a PCV7 record in the IMS DA database. This list was compiled by identifying those who had no event or prescription codes corresponding to pneumococcal vaccination (Read codes listed in Table 1 or ATC code J07A7). A computer generated random sample of 173 apparently unvaccinated children was made and in December $2010 \mathrm{a}$ letter and one page questionnaire, with the child's age, gender, date of birth and patient ID, was sent by IMS-DA to the relevant GP asking them to review the child's health record and report if they had received Prevnar $7^{\circledR}$ vaccine and which of the three doses they had been given. The general practitioner received $£ 15$ for completion of the questionnaire.

Sample size estimation for questionnaire study

The sample size was based on some preliminary work with IMS DA data indicating that of the approximately 18,000 children aged $0-2$ years registered approximately $3600(15 \%)$ did 
not have any coded record of PCV7 immunisation. Assuming that $5 \%$ of these children were immunised but unrecorded, the sample size needed to detect this with 95\% confidence would be 132 patients (OpenEpi, Version 3, open source calculator-SSPropor). We recognised that it was unlikely to get data on every child so the sample was increased to 173 , allowing for a $25 \%$ none-response rate.

\section{Ethical approval}

This protocol for this study was approved by the IMS Independent Scientific and Ethical Advisory Committee on $10^{\text {th }}$ October 2008.

\section{Results}

The IMS DA database identified 15,237 children born in 2008, of these $12,740(83.6 \%)$ had a coded record of PCV7 vaccination (Figure 1). A random sample of 173 was taken from the 2,497 children with no vaccination record. Six children could not be followed up as their practice had stopped contributing data to IMS DA $(n=1)$ and another practice encountered a technical problem $(n=5)$. Out of 167 questionnaires sent to the children's' general practitioners, 111 questionnaires were returned, giving an adjusted response rate of 111/167 (66.5\%). Based on the general practitioners' responses $71 / 111(64.0 \%)$ were fully vaccinated (3 doses of PCV7 given), 3/111 (2.7\%) partially vaccinated (only 1 or 2 doses given), $28 / 111(25.2 \%)$ were unvaccinated and for $9 / 111(8.1 \%)$ their vaccination status was unknown (Figure 1).

Extrapolating these observations from our subsample of 111 children to the IMS DA cohort would mean that an extra 1597 children had been fully vaccinated with PCV7, 68 partially vaccinated, 630 unvaccinated and for 202 children their vaccination status was unknown. Thus the percentage of fully vaccinated children within this cohort becomes $94.1 \%$ (14337/15237), rather than $83.6 \%$.

The worst case scenario would be if all of the children for whom we had no response from their GPs were unprotected. If we adjust for this potential response bias in our estimate, and combine these children with those who were only partially vaccinated or for whom vaccination status was unknown, it yields a minimum vaccination rate of $90.6 \%$.

\section{Discussion}

Of the 111 children with no PCV7 record in the IMS selected data for whom we were able to validate their vaccination status , $71 / 111(64.0 \%)$ of these had in fact been fully vaccinated with PCV7. This indicates that the IMS vaccination data is incomplete and therefore underestimates the vaccine coverage. The uptake rate of PCV7 would appear to be at least $90.6 \%$, and possibly as high as $94.1 \%$, not the $83.6 \%$ originally estimated from IMS DA database.

These higher vaccination rates comply more closely with other sources of data on immunization uptake, including Cover of Vaccination Evaluated Rapidly (COVER). This scheme was established in 1987 to monitor immunization coverage of children aged 1,2 and 5 years of age $^{3}$. COVER statistics are compiled by Public Health England by extracting vaccine coverage data each quarter from all child health information systems throughout the country. COVER national statistical data for 2010-2011 estimated the vaccine (PCV7) coverage reached approximately $94 \%$ children by their first birthday. ${ }^{4}$

Why might there be this degree of under reporting of vaccination uptake in database analysis? One can only speculate, but possible explanations include no access to the patients' electronic health record at the time of immunization, lack of triggers to enter vaccine administration on the electronic health record (for example, the vaccine is available in practice without the need for personalized prescription). The data may also sometimes be entered into the electronic health record as a free text entry rather than Read coded and this 
means it is not recognized in routine searches and retrieval. The fee General Practices are paid for each immunization administered might theoretically incentivize the recording child immunization, but the payment is small, its existence may not be apparent to staff uninvolved with the financial and business aspects of the practice, and it rarely impacts on the income of salaried staff. ${ }^{5,6}$ Problems with the computer system has been highlighted as a barrier to fully implementing pneumococcal immunization programs in a previous study. ${ }^{5}$ Additionally, immunization has never been included as an indicator of quality in the Quality and Outcomes Framework (QOF) scheme which has achieved better documentation and some improved clinical care in other areas of health. ${ }^{7}$

What are the clinical implications of our research? Although this work focused only on PCV7 immunization it is likely to be relevant to all the other childhood immunization programs, including influenza, measles, mumps and rubella (MMR), rotavirus vaccine and meningitis $B$ \& $C$ vaccine. A multifaceted approach is needed to improve the completeness and standardization of the recording of vaccination in the primary care electronic health record. This will include ensuring relevant members of the primary care team, including health visitors and school nurses can, and do, access the child's EHR for timely entry of data. Vigilance is also required to ensure that when vaccines are administered in a setting other than primary care, for example in a hospital setting or private health care facility, that the child's general practitioner is informed so that the information can be entered into the child's EHR. With such changes the completeness of databases derived from the primary care EHR will be improved and IMS DA will be able to offer more valid data that can be used in the evaluation of vaccination coverage and also to investigate the impact of vaccines on specific disease prevention.

\section{Strength \& limitations}

The major strength of our study is that the IMS Disease Analyser uses data broadly representative of the UK population in terms of age and gender, although there is under representation of smaller practices and of practices in Scotland and Northern Ireland ${ }^{2}$. This is the first study to validate immunization statistic estimates from the IMS DA. The limitation of the study is that it focused only on underreporting; no attempt was made to confirm the validity of positive coding of vaccination. A third of GPs did not respond to the enquiry, and there is a risk that there may be responder bias with the practitioners of unvaccinated children being less likely to respond. This was recognised in our calculation of the worst case scenario.

\section{Conclusions}

This validation study has demonstrated that individual patient-level data analyses based on IMS data cannot reliably be employed to investigate the association between PCV7 vaccination and the incidence of pneumococcal infection diseases.

The under reporting of vaccination in IMS data arises because of failure to routinely code all childhood immunizations in the primary care electronic health record, and we need to better understand the challenges encountered in practice.

\section{Acknowledgements}

We thank all general practitioners that have contributed to the study. We also thank Professor Mike Sharland from the Paediatric Infectious Diseases Research Group, St George's University London and Mr. Pete Stephens from IMS for their advice and assistance. 


\section{References}

1. Department of Health. Important changes to the childhood immunisation programme. 2006. PL/CMO/2006/1.

2. Wong I, Murray M. The potential of UK clinical databases in enhancing paediatric medication research. British Journal of Clinical Pharmacology 2005; 59(6):750-755.

3. Public Health England. Cover of Vaccination Evaluated Rapidly (COVER) Implementation Guidance. 2014.

4. NHS Immunisation Statistics, England 2010-11. March 2015

http://www.hscic.gov.uk/catalogue/PUB00244/nhs-immu-stat-eng-2010-2011-rep.pdf

5. Shankar A, Samraj R, Aiyedun V, et al. General practitioners' perceptions on pneumococcal vaccination for children in United Kingdom. Human Vaccine 2009; 5:177-80.

6. Campbell S, Reeves D, Kontopantelis E, et al. Effects of Pay for Performance on the Quality of Primary Care in England. New England Journal of Medicine 2009; 361(4), 368378.

7. Dixon A, Khachatryan A, Wallace A, et al. The Quality and Outcomes Framework (QOF): does it reduce health inequalities? National Institute for Health Research 2011. 
Figure 1: Questionnaire Responses

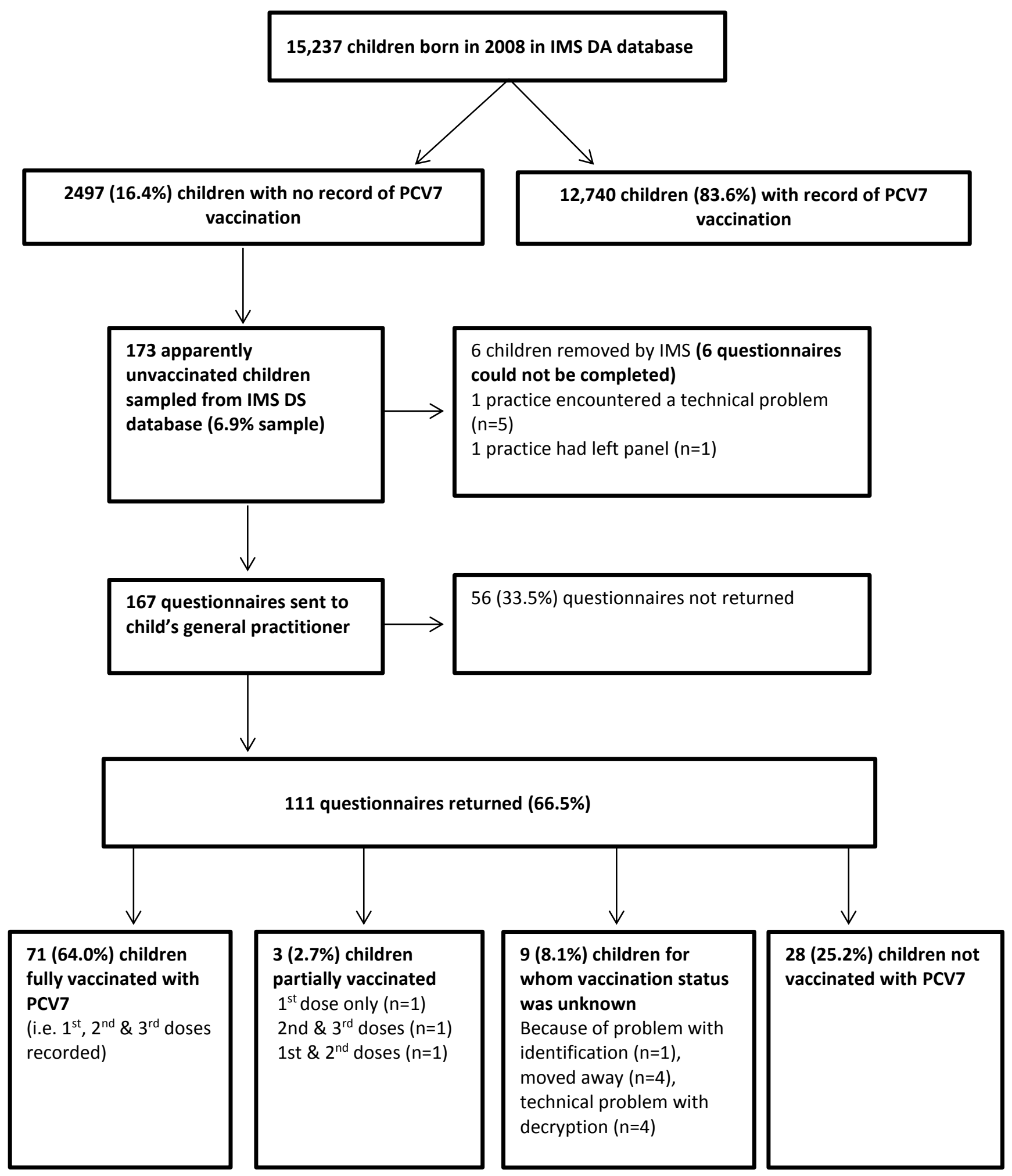


Table 1: Read 4 and Read 5 codes used in IMS DA database search to identify those two year old children who had received pneumococcal vaccination

\begin{tabular}{|c|c|}
\hline READ_4or5_CODE & READ_4or5_TEXT \\
\hline 65720 & $\begin{array}{l}\text { Pneumococcal vaccination } \\
\text { given }\end{array}$ \\
\hline .900. & $\begin{array}{l}\text { Pneumococcal vaccination } \\
\text { admin }\end{array}$ \\
\hline .6572 & Pneumococcal vaccination \\
\hline.$n 4 b$. & Pneumococcal vaccine \\
\hline $.68 \mathrm{Ne}$ & $\begin{array}{l}\text { Consent pneumococcal } \\
\text { vaccine }\end{array}$ \\
\hline $.657 \mathrm{~K}$ & $\begin{array}{l}\text { Booster pneumococcal } \\
\text { vaccinate }\end{array}$ \\
\hline .Q5AC & $\begin{array}{l}\text { Adv reac: pneumococcal } \\
\text { vaccine }\end{array}$ \\
\hline $.657 \mathrm{~N}$ & $\begin{array}{l}\text { 3rd pneumococcal conjug } \\
\text { vaccine }\end{array}$ \\
\hline $.657 \mathrm{M}$ & $\begin{array}{l}\text { 2nd pneumococcal conjug } \\
\text { vaccine }\end{array}$ \\
\hline $.657 \mathrm{~L}$ & $\begin{array}{l}\text { 1st pneumococcal conjug } \\
\text { vaccine }\end{array}$ \\
\hline
\end{tabular}

